\title{
Waveguide analysis for ultrasonic medical instruments (comparison of different methods)
}

\author{
Yuri V. Grigoryev, and Pyae Phyo Aung* \\ Bauman Moscow State Technical University, 105005 Moscow, Russian Federation
}

\begin{abstract}
Transition matrices are derived on the basis of some analytical solutions for a number of waveguides. Initial parameters method is used to analyze the waveguide longitudinal oscillations. This paper contains a comparative analysis of different types of concentrators. The waveguide design scheme presents a straight bar of variable cross-section with different boundary conditions. For given concentrator arrangements, the amplitude axial displacements and longitudinal forces in the ultrasonic medical instrument (UMI) are calculated for a certain excitation frequency. Numerical method for calculation of eigenfrequencies and eigenmodes of the ultrasonic medical instrument (UMI) waveguide is presented. Analysis of the cantilever straight-line concentrator based on the methods of transition matrices and numerical integration by the Runge-Kutta method are under comparison. Sufficient agreement of the results is demonstrated.
\end{abstract}

\section{Eigenfrequencies and eigenmodes of ultrasonic medical instruments determination}

The most widespread waveguides-concentrators with exponential, catenoidal and conical horns, as well as waveguides of constant cross section [1-4]. The cross-sectional area of these waveguides is respectively

$$
\begin{gathered}
A(z)=A_{0} \exp (-2 \beta z) \text { - exponential horn } \\
A(z)=A_{k} \operatorname{ch}^{2}(l-z) \text { - catenoidal horn } \\
A(z)=A_{0}\left(l-\frac{z}{a}\right) \text { - conical horn }
\end{gathered}
$$

Constants $\beta, \gamma$ and $a$ characterize the rate of contraction of the corresponding horn and are determined by the cross-sectional area of the concentrator at $z=0$ and $z=l$, respectively $l$ (the length of the tapering portion (horn)).

$$
\beta=1 / l \ln \sqrt{A_{0} / A_{k}} ; \gamma=1 / l \cdot \operatorname{arcch} \sqrt{A_{0} / A_{k}} ; a=\frac{1}{1-\sqrt{A_{0} / A_{k}}},
$$

Coefficient $g(z)$ depending on the type of contraction will be equal to

\footnotetext{
*Corresponding author: pyaephyo88@mail.ru
} 
- for the exponential contraction $g(z)=-2 \beta$;

- for catenoidal contraction $g(z)=-2 \gamma \cdot \operatorname{th}[\gamma(l-z)]$;

- for the cone $g(z)=2 /(z-a)$.

For the sites of concentrators within which the cross-section area changes exponentially, equation (2) has the form

$$
U^{\prime \prime}-2 \beta u^{\prime}+\alpha^{2} u(z)=0
$$

Solution at $\beta>\alpha$ (steep horn):

$$
u(z)=\exp (\beta z) \cdot\left(C_{1} \mathrm{ch} \alpha_{1} z+C_{2} \mathrm{~s} h \alpha_{1} z\right),
$$

where $\alpha_{1}=\sqrt{\beta^{2}-\alpha^{2}}$

When $\beta<\alpha$ (gentle horn)

$$
s_{1,2}=\beta \pm i \sqrt{\alpha^{2}-\beta^{2}}=\beta \pm i \alpha_{1}, \quad \alpha_{1}=\sqrt{\beta^{2}+\alpha^{2}}
$$

And the solution is

$$
u(z)=\exp (\beta z) \cdot\left(C_{1} \cos \alpha_{2} z+C_{2} \sin \alpha_{1} z\right),
$$

For catenoidal contraction equation (2) has the form:

$$
u^{\prime \prime}(z)-2 \gamma \tanh (l-z) u^{\prime}(z)+\alpha^{2} u(z)=0
$$

It is easy to make sure that the equation can be represented as

$$
[u \cosh \gamma(l-z)]^{\prime \prime}+\left(\alpha^{2}-\gamma^{2}\right) \cosh \gamma(l-z) u(z)=0
$$

And using substitution $u_{0}(z)=u(z) \cosh \gamma(l-z)$, we'll get

$$
u^{\prime \prime}(z)+\left(\alpha^{2}-\gamma^{2}\right) u_{0}(z)=0,
$$

The solution of this equation is

$$
\begin{aligned}
& u(z)=[\cosh \gamma(l-z)]^{-1} \cdot\left(C_{1} \cos \alpha z+C_{2} \sin \alpha z\right) ; \\
& \alpha_{1}=\sqrt{\alpha^{2}-\gamma^{2}}, \alpha>\gamma .
\end{aligned}
$$

For the conical portion of the concentrator, it can be obtained

$$
u^{\prime \prime}(z)+2 /(z-a) \cdot u(z)+\alpha^{2} u(z)=0
$$

Where, $(a)$ is the full length of the cone.

The solution of this equation is

$$
u(z)=\frac{1}{(a-z)}\left(C_{1} \cos \alpha z+C_{2} \sin \alpha z\right) ;
$$

For all of the above cases, the amplitude of the axial force $N(z)$ is 


$$
N(z, t)=N(z) \cos (\omega t)
$$

The eigenvalue problem is solved by the following condition:

$$
\operatorname{det} A\left(\alpha l_{k}\right)=0
$$

To solve the eigenvalue problem for the selected UMI element as a whole, it is convenient to use the matrices variant of the initial parameters method [6-8].

\section{Matrices method of ultrasonic medical instruments calculation}

Consider a waveguide of constant cross-section length $(l)$. The General solution for this case is equation (2) in the form

$$
u(z)=C_{1} \sin \alpha z+C_{2} \cos \alpha z
$$

The amplitude of the axial forces $N(z)$ is

$$
N(z)=E A(z) \alpha\left(C_{1} \sin \alpha z+C_{2} \cos \alpha z\right)
$$

The transition matrices for the conical section will be equal to

$$
A_{\text {con }}=\left[\begin{array}{cc}
\frac{a \cos (\alpha l) \alpha-\sin (\alpha l)}{(a-l) \alpha} & -\frac{a \sin (\alpha l)}{(l-a) E A_{0} \alpha} \\
\frac{E A_{k}\left(-\sin (\alpha l)+a \sin (\alpha l) \alpha^{2} l-a^{2} \sin (\alpha l) \alpha^{2}+\alpha \cos (\alpha k) k\right)}{(a-l)^{2} \alpha} & \frac{A_{k}(a \cos (\alpha l) \alpha-\cos (\alpha l) \alpha l+\sin (\alpha l)}{(-1+a)^{2} A_{0} \alpha}
\end{array}\right]
$$

where $\left(A_{0}\right)$ is the cross-sectional area at the beginning of the section

$N_{d}=\sqrt{A_{0} / A_{k}} ; A_{k}$ - the cross-sectional area at the end of the plot.

The transition matrices for the exponential segment will be equal to

$$
A_{\text {exp }}=\left[\begin{array}{cc}
e^{\beta l}\left(\cos \left(\alpha_{1} l\right)-\frac{\beta \sin \left(\alpha_{1} l\right)}{\alpha_{1}}\right) & \frac{e^{\beta l} \sin \left(\alpha_{1} l\right)}{E A_{0} \alpha_{1}} \\
\frac{e^{-\beta l} E A_{0} \sin \left(\alpha_{1} l\right)\left(\alpha_{1}^{2}-\beta^{2}\right)}{\alpha_{1}} & \frac{e^{-\beta l}\left(-\beta \sin \left(\alpha_{1} l\right)-\alpha \cos \left(\alpha_{1} l\right)\right.}{\alpha_{1}}
\end{array}\right]
$$

The transition matrices for the catenoidal area will be equal to

$$
A_{\text {cat }}=\left[\begin{array}{cc}
\cosh (\gamma l) \cosh \left(\alpha_{1} l\right)-\frac{\sinh (\gamma l) \gamma \sin \left(\alpha_{1} l\right)}{\alpha_{1}} & \frac{\cosh (\gamma l) \sin \left(\alpha_{1} l\right)}{E A_{0} \alpha_{1}} \\
E A_{k}\left(-\cosh (\gamma l) \sin \left(\alpha_{1} l\right) \alpha_{1}-\sinh (\gamma l) \gamma \cos \left(\alpha_{1} l\right)\right) & \frac{A_{k} \cosh (\gamma l) \cos \left(\alpha_{1} l\right)}{A_{0}}
\end{array}\right]
$$

For steep transitions (horns), when $\gamma$ or $\beta$ more than $\alpha$, all trigonometric functions in the transition matrices should be replaced by appropriate hyperbolic (this does not apply to conical horns) [10].

Let's assume that UMI consists of sections, within each of which the law of change of the cross-section area is such that one of the functions (1) can be approximated, and for each of the sections a local coordinate system $O_{i} Z_{i}$ is introduced, the beginning of each of which coincides with the left edge of the corresponding $i$-th section $[5,7,10]$. The solution vector at 
$\mathrm{Z}_{1}=0$ has the form: $\bar{V}(0)=\bar{V}_{0}^{(1)}$ Then the solution at the end of the first section will look like this $V_{k}^{(1)}=A^{(1)} \bar{V}_{0}^{(1)}$, but the strength of the continuity and equilibrium conditions vector of the solution at the beginning of the next section $\bar{V}_{0}^{(2)}$ coincides with $\bar{V}_{k}^{(1)}$ Decision at the end of the second phase: $V_{k}^{(2)}=A^{(2)} \bar{V}_{0}^{(1)}$, but $V_{0}^{(2)}=\bar{V}_{k}^{(1)}$. So $V_{k}^{(2)}=A^{(2)} A^{(1)} \bar{V}_{0}^{(1)}$,

Decision at the end of the $i$-th section:

$$
V_{k}^{(i)}=A^{(i)} A^{(i-1)} \ldots A^{(2)} \bar{V}_{0}^{(1)}
$$

The solution at the end of the n-th section: ( i.e $Z=l=\sum_{i=1}^{n} l_{i}$,., where $l_{i}$ is the length of the $i$-th section, $(l)$ is the total length of the ultrasonic vibrating system) has the form

$$
{\overline{V_{k}}}^{(n)}=B(w) \bar{V}_{0}^{(1)}
$$

where $B(w)=\prod_{i=1}^{n} A^{(i)}$

Eigenfrequencies (resonant lengths) of the UMI are found from the solution of the equation (19), and the boundary conditions on the right and left edges of the interval $[0, l]$ must be satisfied.

We consider different variants of boundary conditions:

1) The left and right edges are free $(\mathrm{N}=0)$, then

$$
\bar{V}_{0}=\left(u_{0}, 0\right) ; \bar{V}_{k}=\left(u_{k}, 0\right),
$$

in this case we have the following frequency equation:

$$
\left(\begin{array}{c}
u_{k} \\
0
\end{array}\right)=\left(\begin{array}{ll}
b_{11} & b_{12} \\
b_{21} & b_{22}
\end{array}\right) \cdot\left(\begin{array}{c}
u_{0} \\
0
\end{array}\right) \text { or } b_{21}(\omega)=0
$$

2) The left edge is free $(\mathrm{N}=0)$, the right one is closed $(\mathrm{u}=0)$, then

$$
\overline{V_{0}}=\left(u_{k}, 0\right) ; \overline{V_{k}}=\left(0, u_{0}\right) ;
$$

in this case we have the following frequency equation:

$$
\left(\begin{array}{c}
0 \\
N_{k}
\end{array}\right)=\left(\begin{array}{ll}
b_{11} & b_{12} \\
b_{21} & b_{22}
\end{array}\right) \cdot\left(\begin{array}{c}
u_{0} \\
0
\end{array}\right) \text { or } \quad b_{1}(\omega)=0
$$

\section{Analytical solution}

\subsection{The solution of the transition matrices}

Let's take a waveguide consisting of cylindrical, conical and exponential sections, made of titanium alloy BT- 5 [9]. 


$$
A(z)=\mid \begin{gathered}
A_{c y l} \text { if } 0 \leq z \leq L_{c y l} \\
A_{c y l}\left(1-\frac{z-L_{c y l}}{a}\right)^{2} i f L_{c y l} \leq z \leq L_{c y l}+L_{c o n} \\
A_{c o n} e^{-2 \beta\left[z-\left(L_{c y l}+L_{c o n}\right)\right]} i f L_{c y l}+L_{c o n} \leq z
\end{gathered}
$$

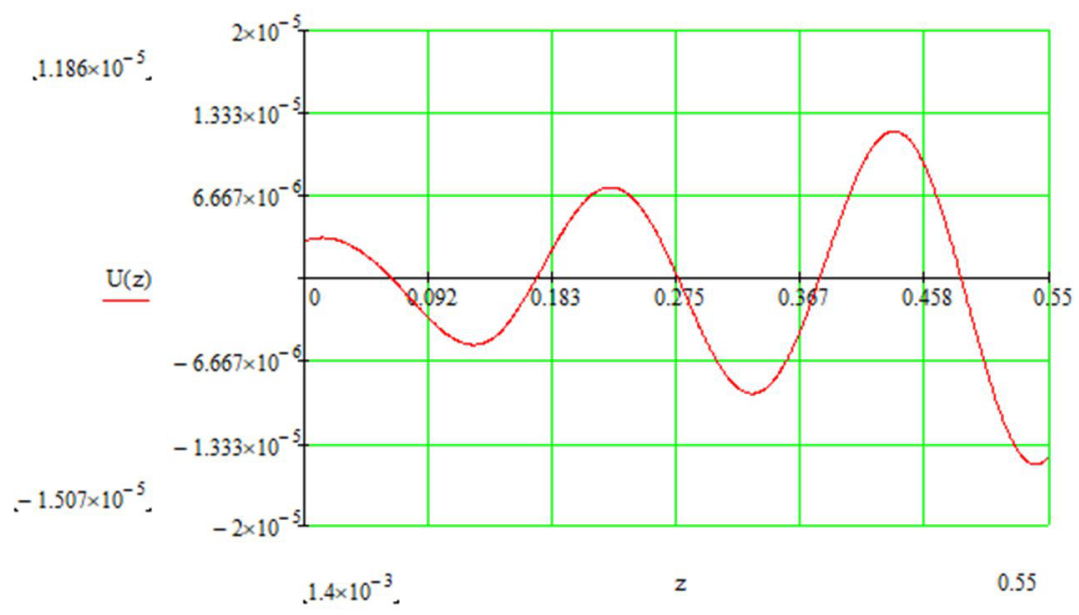

Fig.1. Longitudinal displacements of the waveguide.

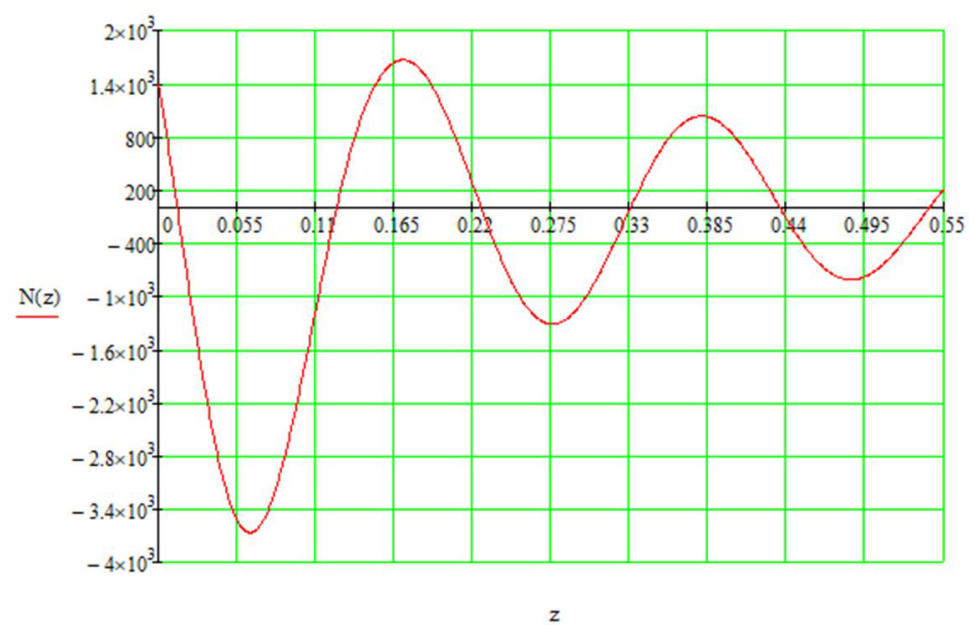

Fig.2. The normal force in the cross-sections of the waveguide.

\section{Solution of the equation by Runge-Kutta}

The Runge-Kutta method is a method of iterative approximate integration of differential equations. We solve our problem using the fourth order Runge-Kutta method [1].

Differential equation of oscillations is

$$
U_{0}(z)^{\prime \prime}+g(z) U_{0}(z)^{\prime}+\alpha^{2} U_{0}(z)=0
$$


We transform the second-order differential equation into a system of first-order differential equations. To do this, make a replacement:

$$
\left\{\begin{array}{c}
\frac{d U(z)}{d z}=Y(z) \\
\frac{d Y(z)}{d z}=-g(z) Y(z)-\alpha^{2} U_{0}(z)
\end{array}\right.
$$

Vector of initial conditions: $Y_{1}=\left(\begin{array}{l}u_{1} \\ C_{1}\end{array}\right)$, where $\mathrm{u}_{1}=3 \mu \mathrm{m}$

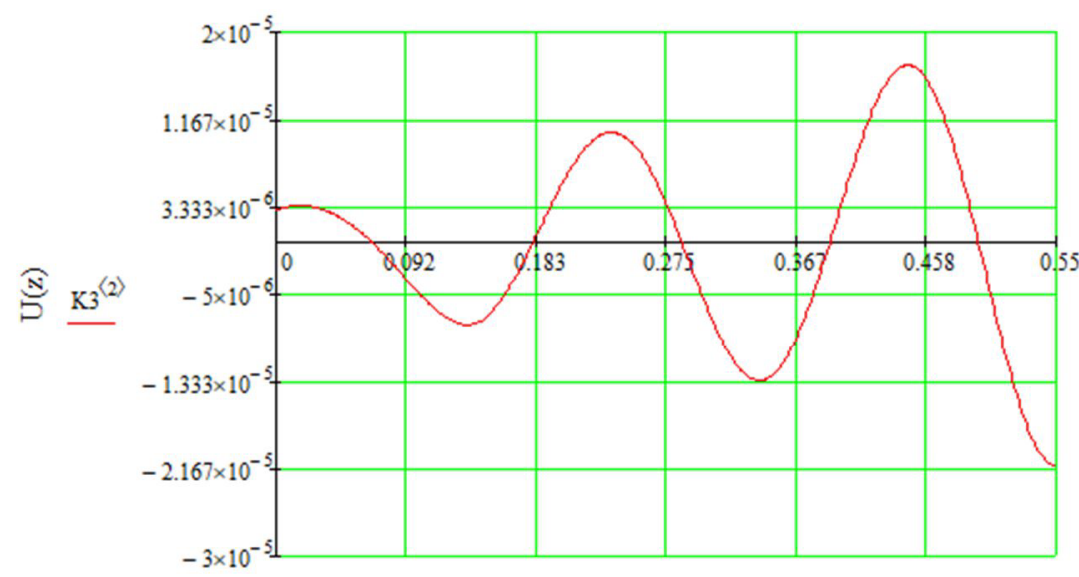

$$
\mathrm{K} 3^{\langle 1\rangle}
$$

Z

Fig.3. Longitudinal displacements of the waveguide.

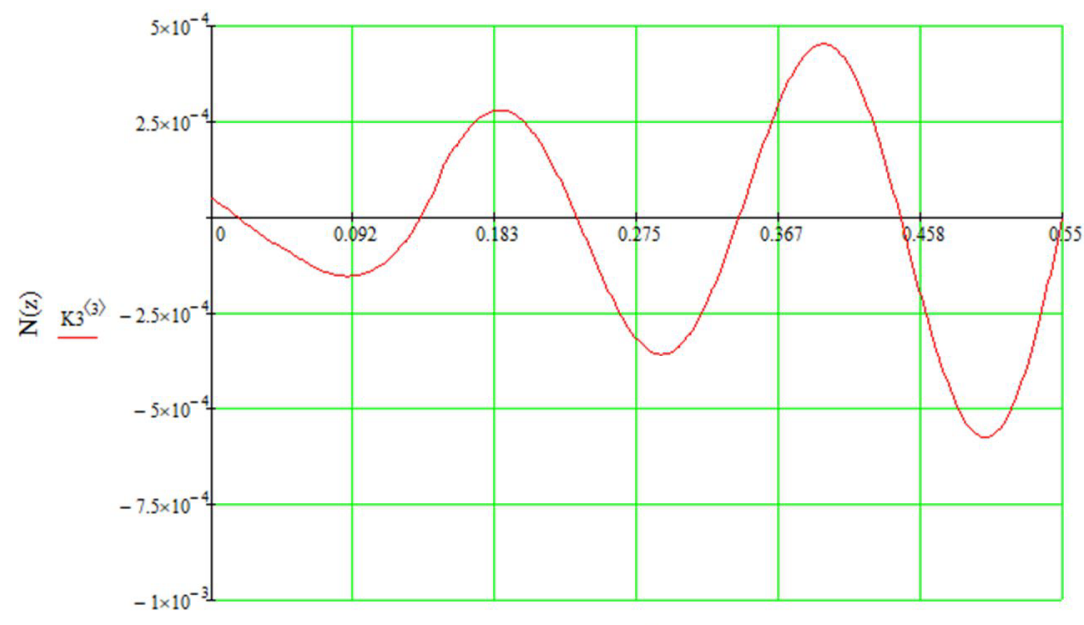

$$
\mathrm{K}^{\left\langle{ }^{\langle 1\rangle}\right.}
$$

Fig.4. The normal force in the cross-sections of the waveguide. 


\section{Comparison of transition matrices and Runge-Kutta methods}

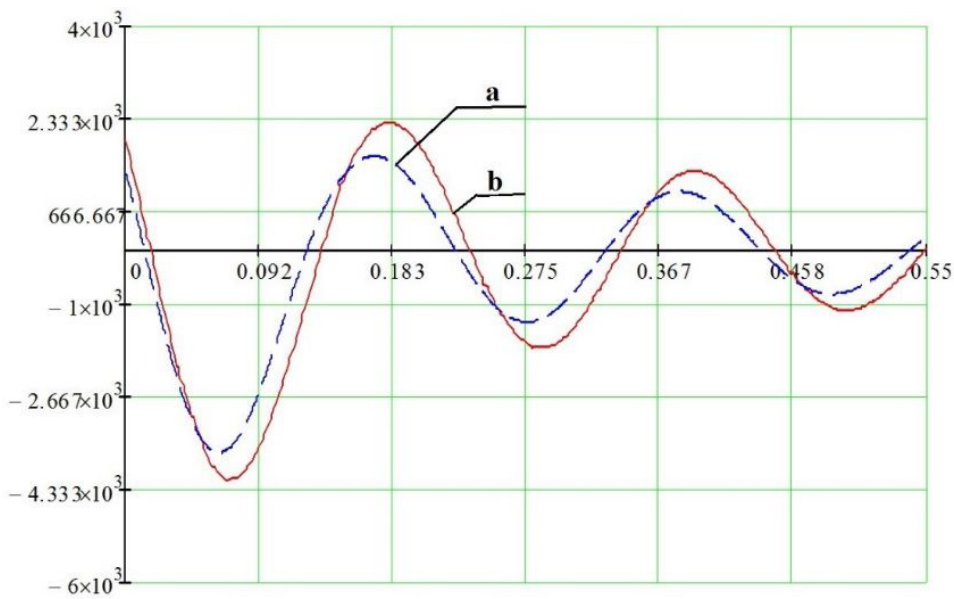

Fig.5. The longitudinal displacement of the sections of the waveguide. a - transition matrices method, b - Runge-Kutta method

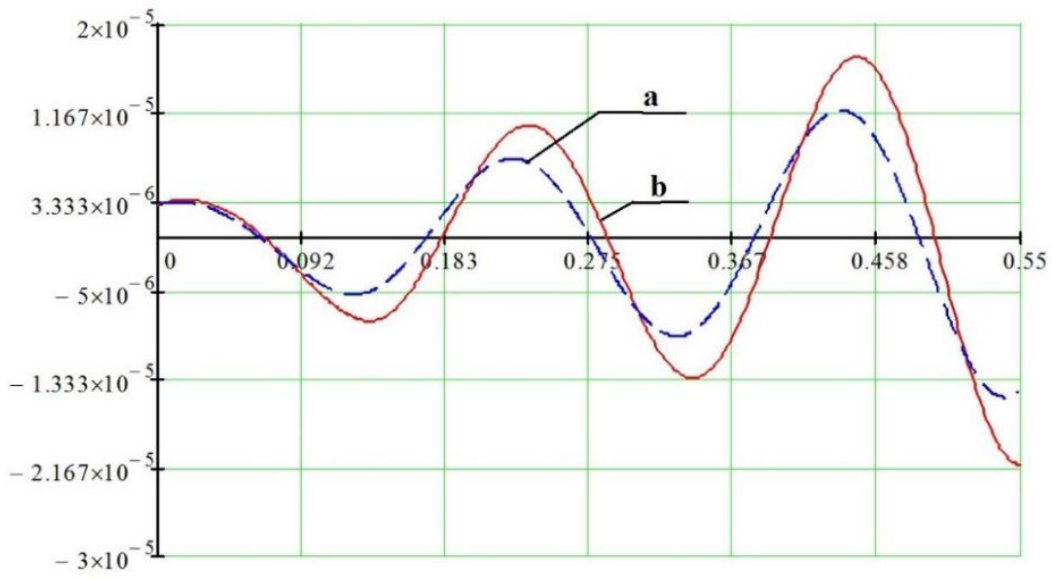

Fig.6. The normal force in the cross-sections of the waveguide. a - transition matrices method, b Runge-Kutta method.

Comparison of the results (Fig. 5, Fig.6) shows their practical coincidence. The obtained solutions are close with acceptable accuracy, which allows for practical calculations application of the transition matrices method, which gives a large gain in time.

\section{References}

1. V. L. Biderman, Theory of mechanical vibrations (Higher School, Moscow, 1980)

2. S. E. Kvashnin, Trudy of the BMSTU, 457, 144 (1986)

3. S. E. Kvashnin, Theory, calculation and design of medical equipment: Methodical instructions (BMSTU, Moscow, 1987)

4. G. A. Nikolaev, V. I. loshilov, Ultrasonic technology in surgery (Medicine, Moscow, 1980)

5. R. Baldaev, Application of ultrasound (Technosphere, Moscow, 2006)

6. V. A. Shutilov, Basic physics of ultrasound (SPBU, Saint-Petersburg, 1980) 
7. B. A. Agranat, Fundamentals of physics and technique of ultrasound (Higher school, Moscow, 1987)

8. E. Kikuchi, Ultrasonic transducers (Mir, Moscow, 1972)

9. A. I. Markov, Ultrasonic processing of materials (Mashinostroenie, Moscow, 1980)

10. V. N. Hmelev, Elect. J. "Technical acoustics", 26, 1-12 (2005) 